\title{
Técnicas de arada en la provincia de Zamora en la Edad Media
}

Hace unos años, con motivo del Homenaje que la Revista de Dialectologia y Tradiciones Populares ofreció a Concha Casado, aportamos un breve artículo en el que hacíamos una lectura etnohistórica de los fueros de la provincia de León ${ }^{1}$. Hoy volvemos sobre el tema en esta nota, abordando un aspecto puntual del mismo y recurriendo a fueros del territorio de la actual povincia de Zamora ${ }^{2}$.

Vamos a tratar sobre las labores que recibía el barbecho en el cultivo de los cereales, recogiendo, de forma tangencial, información relativa a la fuerza de tiro empleada en las faenas de arada y a ciertos usos del arado en cultivos no cerealísticos. Para una mayor claridad consideramos útil reunir en un Apéndice los textos a los que se hace alusión a lo largo de este artículo, ordenados cronológicamente.

\section{EL BARBECHO}

Quizá sea conveniente comenzar por definir lo que es el barbecho, dado que, a veces, la idea sobre el significado de este término y de lo que implica no está expresada claramente. En una rotación bienal de cultivos, el barbecho es la tierra no sembrada que, con posterioridad a la siega, recibe una serie de labores que la preparan para la siguiente siembra. F. Sigaut ha llamado la atención repetidamente sobre este tema, en $1972{ }^{3}$ demostró que lo característico del barbecho son los trabajos que recibe la tierra y no el hecho de «descansar». Esta idea, extendida tanto entre el mundo académico como en el mundo rural, no está realmente vinculada a lo que se deduce del aná-

1 José Luis Mingote Calderón, «Aperos y faenas agrícolas en los fueros de la provincia de León», RDTP, XLIII (1988), 411-428, 7 figs.

2 Al igual que los leoneses, han sido recopilados por Justiniano RoDRíGuEZ FERNÁNDEZ, Los fueros locales de la provincia de Zamora (Salamanca: Junta de Castilla y León. Consejería de Cultura y Bienestar Social, 1990), 412 pp.

3 F. Sigaut, «Les conditions d'apparition de la charrue. Contribution à l'étude des techniques de travail du sol dans les anciens systèmes de culture», Journal d'Agriculture Tropicale et Botanique Appliquée, 10-11 (1972), 442-478. 
lisis etimológico del término. Las voces que designan esta realidad, en francés, inglés, alemán, español, portugués... indican todas lo mismo: relación con el trabajo de la tierra ${ }^{4}$. Para Sigaut, barbecho se podría definir de la siguiente manera:

La jachère èst l'ensemble de labours de printemps et d'été par lesquels on ameublit et on nettoie une terre destinée à être ensemencée en céréales à l'automne suivant. On appelle aussi jachère les terres qui sont en train de recevoir cette préparation ${ }^{5}$.

El confusionismo procede, en otras ocasiones, de la adjetivación del término. Por no alejarnos del mundo medieval, vamos a aludir a las ideas que expone L. Bolens en su conocido trabajo sobre los agrónomos de al-Andalus, algunas de las cuales están en abierta oposición con las expresadas por F. Sigaut. Distingue la autora franco-suiza el barbecho cultivado, el trabajado y el desnudo, nue, al hablar de los cultivos con sistema de rotación trienal ${ }^{6}$. El primer tipo se daría en una tierra que recibe abono pero no riego, implicaría el cultivo primaveral de una planta de ciclo corto - tremesina- o de leguminosas y estaría reemplazando al barbecho (suponemos que al trabajado). El segundo encajaría con la definición expresada al comienzo de este apartado y se daría en campos no regados ni abonados. Por último, el barbecho desnudo se daría en tierras de mediana calidad, dedicadas preferentemente al cultivo del trigo, y supondría una tierra no trabajada.

En nuestra opinión, no debe utilizarse el término barbecho para aludir a realidades diferentes del concepto definido anteriormente. Desde el momento en que una tierra está sembrada deja de estar en barbecho, con lo cual el empleo de «barbecho cultivado» es un contrasentido ${ }^{7}$. Tampoco puede usarse para designar otra realidad que tiene nombre propio. En una rotación trienal, la tierra que no recibe ni simiente ni trabajos preparatorios se denomina baldío, erial o posío, según las zonas; término que es empleado por L. Bolens unas páginas más adelante en su correcta acepción.

4 F. Sigaut, op. cit. en nota 3, pp. 455-456.

5 F. Sigaut, op. cit. en nota 3, p. 457. Con posterioridad a este trabajo ha vuelto sobre el tema en: «La jachère en Écosse au XviIr siécle: phase ultime de l'expansion d'une technique», Études Rurales, 57 (1975), 89-105; «Quelques notions de base en matière de travail du sol dans les anciennes agricultures européennes», Journal d'Agriculture Tropicale et Botanique Apliquée, 24, 2-3 (1977), 139-168, y «La naissance du machinisme agricole moderne», Antropologie et Sociétés, 13, 2 (1989), 79-101.

6 Lucie Bolens, Agronomes andalous du Moyen-Age (Genève. Paris: Librairie Droz, 1981), 305 pp. en concreto en la p. 125.

7 Si al término cultivado se le da el valor de sembrado y no, simplemente, el de trabajado. 
Frente a esta disparidad de criterios, vamos a recurrir a un texto de autor andalusí, toledano por más señas, escrito en el siglo XI, donde se explica con absoluta claridad qué implica este concepto. Ibn Başșāl, en su Libro de Agricultura habla de la necesidad de recurrir al barbecho en los terrenos que no pueden ser abonados con estiércol debido a su gran tamaño. El texto dice:

«... pero en la tierra grande y ancha no es hacedero [el estercolarla] y en su lugar habremos de acudir al barbecho, o sea, a labrar la tierra y hacer que la parte superior de ella quede abajo, y repetiremos esta vuelta diversas veces. Empezaremos esta operación desde mediados de enero, por todo febrero, hasta mediados de marzo, o bien hasta principios de este mes; luego se volverá a arar y a romper, con una buena labor, hasta mediados de abril o hasta las proximidades de mayo. A fines de mayo se reiterará aquella labor y se dejará la tierra a merced del intenso calor que llegará, de modo que ella se homogeneice, se haga delgada y se vayan sus humores. Si lloviere sobre ella en el mes de junio y se embebiera asaz de agua, la araremos por cuarta vez: luego la dejaremos descansar, sin rebasar las cuatro labores o aun las dos labores si la tierra fuera de buena calidad, pues la cebada posee humedad en grado sumo y se adapta bien al aire y a la tierra, de lo cual resulta su ventaja» ${ }^{8}$.

Los editores de este texto llaman la atención sobre el hecho de que, en la traducción que se hizo al castellano de este tratado, a finales del siglo XIII o comienzos del XIV, se incluya el trigo junto a la cebada, a la hora de especificar las técnicas de preparación del suelo ${ }^{9}$.

8 IBN BAȘSALl, Libro de Agricultura. Editado, traducido y anotado por José M." MillaS VAllicrosa y Mohamed Aziman (Tetuán: Instituto Muley el-Hasan, 1955), $231+$ 182 pp., en pp. 62-63.

9 El texto copiado corresponde a un resumen breve de un tratado más amplio, actualmente desparecido. La traducción medieval del mismo dice: Mas la tierra que grant $e$ ancha non le abonda esto. E en logar de estiercol deuenle baruechar $e$ atar [sic, por arar] e trastornar lo deyuso a suso vna en pos otra. E comiençe esto en enero mediado $e$ abran la tierra e trastornenla desde mediado enero a todo febrero. E la meytat de março. E despues tornen a arar aquello arado e rrompan la tierra e vinen la de buena lauor fasta abril mediado o açerca de mayo. E trastornen la terçera vegada fasta mayo salido. E denle vagar a la grant calentura que se buelua vna con otra e se faga delgada. E pierda los malos bumores. E si llouiere sobre ella en el mes de junio fasta ques farte bien dagua aren la quarta vegada. E dexenla estar e non la aren de quatro veses adelante. E esto fagan a la tierra do siembran el trigo sennalada mente mas la tierra $o$ sienbren la ca cunple que la labren dos veses quando fuere buena tierra ca la çeuada a en ella bumidat fuerte $e$ auienese bien con la tierra e con el ayre. Lo recoge José María Mrillas Valuicrosa, «La traducción castellana del "Tratado de Agricultura" de Ibn Bașșāl», Al-Andalus, XIII, 2 (1948), 347-430, en p. 368. 
Otro autor andalusí, el sevillano Ibn al-'Awwām (siglos XII-XIII), alude repetidamente a la importancia de dar varias rejas. Su descripción es, a veces, confusa. No obstante, indica con toda claridad - siguiendo a Ibn Haŷŷây - que la multiplicación de labores tiene un efecto similar a la siembra de legumbres, al abonado y a dejarla descansar «sin voltear, ni sembrar» ${ }^{10}$. Este autor da como procedente de la Agricultura nabatea, del oriental Ibn Wahšsiyya (siglo IX), un texto que coincide parcialmente con el citado de Ibn Bașșāl. Escribe Ibn al-'Awwām:

... el Autor de la Agricultura Nabathea dice, que se voltee a tierra con el instrumento acostumbrado para que quede encima la parte que está debaxo; porque habiendo en esta última xugo, frialdad y humedad, y en la de la superficie sequedad y calor, quando volteada de arriba abaxo, y de abaxo arriba se mezcla entre si, queda aquella tierra en un justo temperamento y de buena calidad; cuyo mejoramiento llega á grado superior despues de binada y terciada [ó despues de la segunda ó tercera reja] ${ }^{11}$.

Para proseguir unas páginas más adelante, con dos fragmentos que retoman el texto del toledano, explicando el porqué de la dificultad de estercolar un campo grande:

Mas no es posible estercolar un terreno grande; fuera de que habiendo en él estiercol que no está bien podrido, la gran copia [de la simiente] de las yerbas estrecharía y sufocaría lo que en ella se sembrase; por lo que en lugar de esto será mejor darle primera, segunda y tercera reja para que el sol pueda penetrarla, y el agua páre en ella, y se le arranque la yerba que se alimentaba de su xugo. Así, siendo mas fácil y posible al hombre arar que estercolar, han preferido los agricultores por esta causa una perfecta manera de labor de experimentada utilidad que llaman de vuelta; y es entre ellos proverbio, que el que no voltea, no ara ${ }^{12}$.

10 Abu Zacaria İahia Aben Mohamed Ben Ahmed EBN EL AwAM, Libro de agricultura. Su autor El doctor excelente... Traducido al castellano y anotado por Josef Antonio Banqueri... (Madrid: Ministerio de Agricultura, Pesca y Alimentación, 1988), 2 vols. de $46+698$ y 756 pp., respectivamente. Es una reproducción facsímil de la edición de 1802, e incluye una introducción de J. Esteban Hernández Bermejo y E. García Sánchez. El texto citado está en pp. 1-2 del tomo II.

11 Op. cit. en nota anterior, p. 4 del tomo II. Hemos conservado la ortografía original. De Ibn Başsāl se ha resaltado su falta de dependencia de la tradición agronómica anterior y el que sus conocimientos están basados en la experimentación; cf. Lucie Bolens, op. cit. en nota 6, p. 25.

12 Op. cit. en nota anterior, p. 7 del tomo II. 
y los momentos en los que se deben dar las labores:

Si hubiere [alguna tierra] que haya de sembrarse al año siguiente, se empezará á arar desde mediados de enero, que es el primer tiempo de esto y el mas ventajoso. La vuelta, que [además de esta] se le ha de dar en febrero, ha de ser menos [profunda], y ménos todavía que las dos la que ha de dársele en marzo. El ultimo tiempo de hacer semejante labor es quando empieza el calor en fines de mayo; entre cuyos dos tiempos se bina de vuelta con otra reja hácia mediado ó fin de abril, con otra se tercia hácia fin de mayo; y si es posible, también se quartéa, empezando por ararla en enero, como hemos dicho [...] Si la tercera reja de vuelta se hubiese dado despues de las lluvias, será muy bueno dar quarta ántes de sembrar; a no ser cebada, pues esta tiene bastante con una labor menos ${ }^{13}$.

Podría parecer, a primera vista, que no tiene mucho sentido comenzar con alusiones a agrónomos andalusíes si se va a hablar de textos cristianos de repoblación, pero no es así. Las concomitancias entre el texto de Ibn Bașşāl y los zamoranos que veremos más adelante son palpables y, como es sabido, la ciudad de Zamora fue repoblada en el siglo X por mozárabes toledanos, junto a otros procedentes de Coria o Mérida y grupos de otros ámbitos culturales, documentándose la presencia de comunidades de este origen en varias localidades de la provincia. En los siglos XI y XII es posible constatar todavía menciones de pobladores de origen mozárabe en la capital ${ }^{14}$, pudiendo existir, por tanto, cierta relación entre ambos contextos.

Los datos zamoranos adquieren importancia si los comparamos con los que, en su día, expusieron Ch. Parain y G. Duby en sus ya clásicos trabajos sobre el mundo rural medieval. El primer autor repasa, fundamentalmente, las opiniones de los textos de los agraristas greco-romanos, destacando tres modelos: para Varron y Jenofonte se deben dar dos rejas preparatorias antes de la siembra, Columela y Paladio propugnan que se den tres $\mathrm{y}$, finalmente, Plinio indica que se puede llegar a cinco e, incluso, a nueve. La Edad Media, en opinión del investigador francés, debió inclinarse por el esquema propuesto por Columela y Paladio, «al menos en las explotaciones mejor organizadas» ${ }^{15}$, dependiendo el aumento o disminución del número de rejas de factortes económicos o de tradiciones y costumbres de

13 Op. cit. en nota anterior, pp. 9-10 del tomo II.

14 Puede verse el trabajo de Armando REPRESA, «Génesis y evolución urbana de la Zamora medieval», Hispania, 122 (1972), 525-545, en concreto las pp. 526, 527 y 529 .

15 Charles PARAIN, «Evolution des techniques agricoles au Moyen Age», en The Agrarian Life of the Middle Ages (Cambridge: Cambridge University Press, ${ }^{21966)}$ y en Outils, ethnies y developpement bistorique (Paris: Éditions Sociales. Terrains, 1979), pp. 47-127, por donde se cita. Las ideas expuestas están en pp. 51-52. 
carácter local. Las cuatro rejas se documentarían en la Alta Normandía en el siglo XV, suponiendo un avance técnico respecto a la realidad circundante de la época ${ }^{16}$.

Por su parte, G. Duby anota la existencia de dos rejas en Francia en los siglos IX y X en rotaciones trienales ${ }^{17}$. En los comienzos del siglo XIV, en los dominios de Thierry d'Hireçon, en el Artois, aparecen documentadas las cuatro rejas, mientras que en los contratos de aparcería del Languedoc durante los siglos XIV y XV se menciona la obligación de dar seis o siete rejas preparatorias previas a la siembra ${ }^{18}$.

Las cronologías que aportan los dos autores franceses, respecto a las cuatro rejas, se adelantan considerablemente en nuestro país, donde aparecen en el siglo XI -en los textos zamoranos y andalusíes- y en el siglo XIII en Segovia ${ }^{19}$. En las propiedades del Cabildo segoviano se detalla el número de labores que se deben dar en el barbecho de determinados cultivos de cereales, siendo variada la gama de posibilidades, pudiendo obligar a dar 3 ó 4 rejas, de 2 a 4,1 ó 2, ó de 2 a $5^{20}$. En nuestro trabajo sobre la provincia de León, ya citado ${ }^{21}$, la variedad de situaciones es amplia sin que se refleje ninguna tendencia clara. Así, podemos encontrar desde una reja en Oteruelo (1417), donde sólo se cita el arelvar y el sembrar, hasta las tres de Santo Tirso y Castrillino (1208) - relevar, binnar y terciar-, pasando por las dos de Santa Marinica de Órbigo (1198) o de Algadefe y Santa Marina (1284).

Quizá una búsqueda más intensiva en la bibliografía medieval europea, dada la proliferación de estudios a raíz de la obra de Duby, llevaría a ade-

16 Op. cit. en nota anterior, p. 86. Durante el siglo XIII los agrónomos británicos habían defendido el uso de las tres labores, situación que se generalizará en las grandes explotaciones en fechas posteriores, según $\mathrm{Ch}$. Parain.

17 Georges DubY, Economía y vida campesina en el occidente medieval (Barcelona: Ediciones Península, $\left.{ }^{2} 1973\right), 550$ pp.; la primera edición francesa es de 1962. Lo menciona en p. 37.

18 Op. cit. en nota anterior, p. 145 y nota 48 en la misma página.

19 Propiedades del Cabildo segoviano. Sistemas de cultivo y modos de explotación de la tierra a fines del siglo XIII. Estudios realizados por Ángel García Sanz, José Luis Martín, José Antonio Pascual y Vicente Pérez Moreda (Salamanca: Ediciones de la Universidad de Salamanca, 1981), 184 pp. La transcripción del texto la realiza este autor en las pp. 109-167. Dada la riqueza de datos que ofrece este documento tenemos pensado realizar un trabajo sobre los aspectos relacionados con faenas y aperos agrícolas.

20 Op. cit. en nota anterior, pp. 110 -Domingo Mínguez en Otero Redondo y Matute-, 133 -el arcediano de Cuéllar en Carbonero de Liedos-, 137 -Domingo Mínguez en Matute-. La variedad se da, incluso, en los arrendamientos de una misma persona.

21 En nota 1, pp. 418-420. 
lantar en el resto de Europa las fechas recogidas por este autor y por Parain.

\section{LAS LABORES EN EL BARBECHO SEGÚN LOS DATOS ZAMORANOS}

Los fueros zamoranos en los que se mencionan las faenas relativas al barbecho, con diferente grado de precisión, son los siguientes: Zamora (anterior a 1062), Santa Cristina (1062 y 1226), Valle (1094), Fuentesaúco (1133), Fresno de la Ribera (1146), Villalonso (1147), Fradejas (1148 y 1232), Almaraz de Duero (1175, 1194-1217 y una concordia de 1224), Bamba (1224) y Cañizo (1234) (Fig. 1) ${ }^{22}$; localidades que, en su mayoría, están situadas en las cuencas de los ríos Duero y Valderaduey. Su marco cronológico, como se ve, abarca algo más de siglo y medio y, en cuanto a los otorgantes, son variados, destacando el obispado de Zamora -a través de tres de sus obispos- en lo relativo a la cantidad de documentos ${ }^{23}$.

En ellos se aprecia, como en otros muchos textos medievales, la diferenciación socioeconómica basada en la posesión de animales de tiro o en su carencia, lo que atañe al barbecho, en algún caso, como vamos a ver. Esta diferencia se documenta en Venialbo (1126): de ipso labore que ibi laboraverint cum bove vel cum azada; Fresno de la Ribera: Et de quantum laboraverint cum boves et cum azada; Villalonso: Et qui non babuerit bovem neque asinum faciat secundum possibilitatem suam; Fradejas: Et de omnibus qui laboraverint cum boves et cum axada. En los fueros de Bamba y Cañizo esta disparidad incide en los trabajos relativos al barbecho. En Bamba, la persona que no tiene bueyes queda a disposición del merino del obispo de Zamora para hacer cualquier trabajo: Si vero bovem non habuerit, faciant sernam cum suo corpore ubi maiorinus Episcopi mandaverit in Bamba vel in suo termino. Con mayor claridad, en Cañizo esta diferencia económica implica que quien no tenga bueyes debe acudir a trabajar la

22 Hemos incluido algunos textos en los que solamente se citan aspectos relacionados con la fuerza de tiro o con el empleo del arado en cultivos no cerealísticos, como los de Venialbo o Manganeses de Lampreana,

23 Para una mayor información se debe consultar el libro de J. Rodríguez Fernández, citado en la nota 2. Sobre los vasallos de los obispos zamoranos publicó dos trabajos José Luis MARTiN RodrfgueZ, Campesinos vasallos de la iglesia de Zamora en los siglos XII-XIII (Zamora: Universidad de Salamanca. Colegio Universitario de Zamora, 1977), 44 pp. y Campesinos vasallos del obispo Suero de Zamora (1254-1286) (Salamanca: Universidad de Salamanca. Colegio Universitario de Zamora, 1981), 31 pp. El primero de ellos está editado, asimismo, en Estudis d'Història Agrària, 1 (1978), 85-97, sin el apéndice documental. 


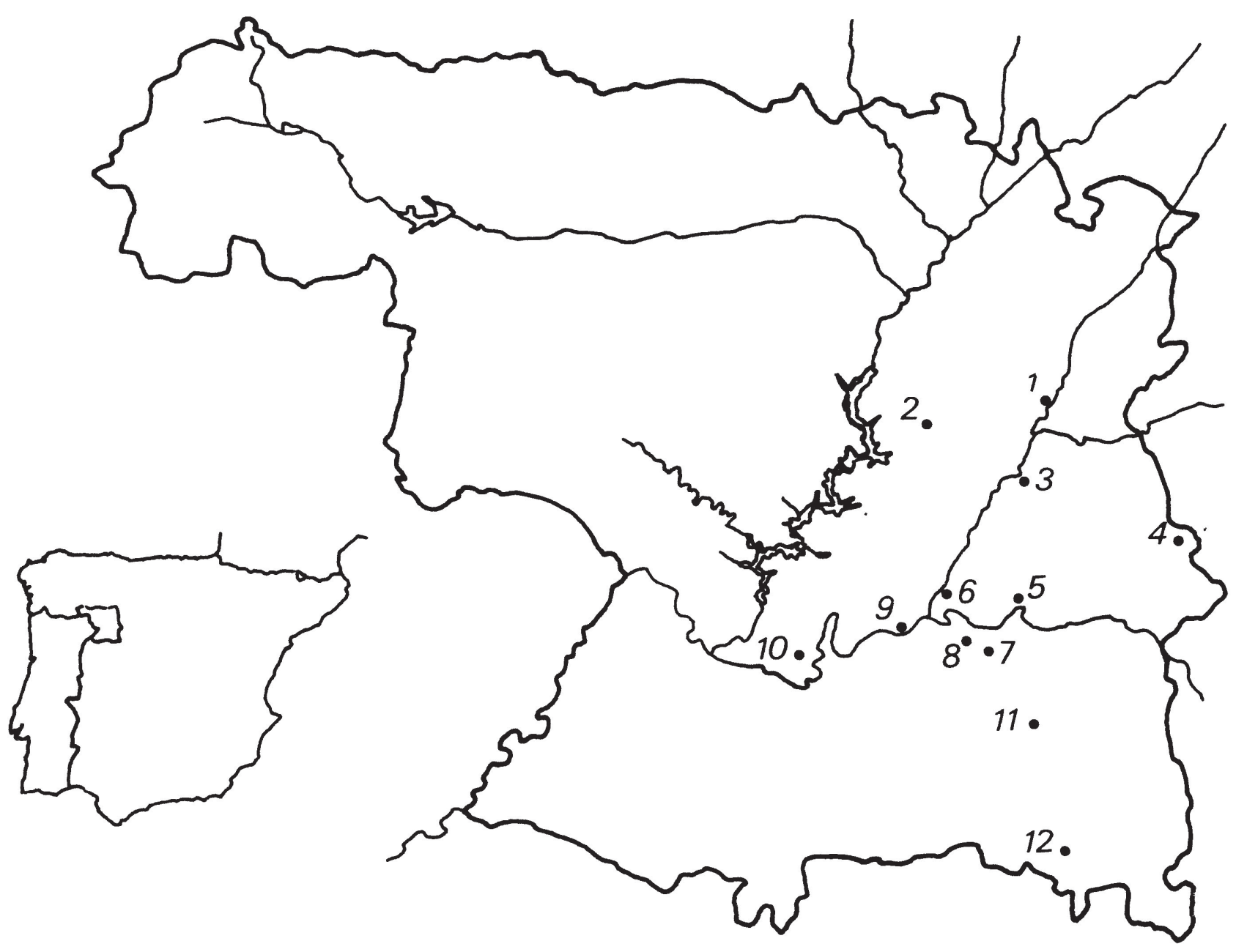

FIG. 1. Localización de los lugares recogidos en el Apéndice documental. 1, Cañizo; 2, Manganeses de Lampreana; 3, Fradejas; 4, Villalonso; 5, Fresno de la Ribera; 6, Santa Cristina; 7, Bamba; 8, Valle; 9, Zamora; 10, Almaraz de Duero; 11, Venialbo, y 12, Fuentesaúco. No se incluye Villamor de los Escuderos. 
viña con su legón o ir a donde se le envíe: Non haventes boves vadant ad vineam cum suis legonibus, vel ad aliud servitium quod ex parte nostra illis fuerint commendatum ${ }^{24}$.

El empleo de bueyes como fuerza de tiro aparece vinculado de forma expresa, en estos textos, a las labores de arada en bastantes casos. En $\mathrm{Za}$ mora, son los animales usados tanto en la arada como para tirar del carro y trillar, ya que en el epígrafe dedicado a su venta se especifica que: se buenos fueren darada, tengalos [el comprador] e enno tienpo de las miesses, pruevelo al carro e enna trilla. En Venialbo se alude a ubi arant nostros boves, mientras que en Villalonso, tras citar que se hagan IIII ieras in arada, se especifica que qui non habuerit bove det suo asino para hacer labores de acarreo. El concierto entre la iglesia de Zamora y Guterre Pardo, sobre unas tierras en Almaraz de Duero, le obliga a este último a hacer sernas con quatuor iugis boum, sernas que son: ralbar, binar y sembrar. En Bamba se obliga a ir a las mismas sernas que en el caso precedente cum melioribus bobus, mientras que en Cañizo se cita a Omnes haventes bobes obligándoles a ralbar dos días cum suis bobis, a la vez que se castiga a quienes lleven jugum vel aratrum vel comerem [(sic) vomerem] malum ${ }^{25}$. En Santa Cristina, cum quantis ingis boum laboraverint, cum tantis faciant sernas supradictas, es decir, ralbar, binar y sembrar. Finalmente, en Manganeses de Lampreana, quien tuviese posesiones por valor de treinta maravedís debe poner un boy con otro omne que ponga otro en arada ${ }^{26}$.

24 La utilización del legón en la viña no implica necesariamente la ausencia de labores de arado en la misma, como se ve en el concierto entre el obispo de Zamora y el concejo de Manganeses de Lampreana (siglo XIII), donde se obliga a todo poseedor de viñedos a darles cuatro labores: escavar et podar, arar et acobondrar. Ignoramos si en Cañizo se producía esta dualidad.

25 El hecho de incluir cláusulas sobre la forma de hacer el trabajo o sobre la calidad de los aperos y los animales, ha sido relacionado con la presión de los señores feudales sobre sus vasallos y la existencia de conflictos entre ambas partes ante la implantación del modelo feudal. Ha sido Javier PÉREZ-EMBID WAMBA, quien ha sugerido tal idea, apoyándose precisamente en este texto de Cañizo, en su trabajo «Violencias y luchas campesinas en el marco de los dominios cistercienses castellanos y leoneses en la Edad Media», en El pasado bistórico de Castilla y León. Volumen 1. Edad Media (Burgos: Junta de Castilla y León. Consejería de Educación y Cultura, 1983), pp. 161178, en concreto en las pp. 163-164. Hemos recogido estas cláusulas en nuestro Doc. 12. Etnológicamente, resulta llamativa la frase en la que castiga a quien no haga bien el trabajo vel rixas permoverit, ya que nos conduce a ese ambiente festivo de toda reunión concejil en la que, normalmente, abunda el vino.

${ }^{26}$ Para estas fechas, ya hay textos burgaleses en los que se aprecia la convivencia de bueyes y bestias - mulos/as o asnos- en faenas de arada y trilla. El fuero de Nuez de Abajo, de 1237, es claro en este sentido: E uos ke nos aiudedes sienpre dos dias 
Centrándonos ya en las labores que recibe el barbecho, hay que señalar que los términos empleados en estos textos para designar las aradas que deben darse a la tierra, se continúan utilizando en la actualidad en la provincia. Si acudimos a estudios lingüísticos sobre la zona oriental de la provincia - incluye las localidades de Manganeses de Lampreana y Venialbo-, o las zonas de Aliste y Sayago, vemos que relvar/reblar/rebrar y bimar/binar se usan en la primera área citada ${ }^{27}$, relvar/relvare y bimar/bimare, en la segunda ${ }^{28}$ y relvar y vimar en la tercera ${ }^{29}$.

En los textos conservados de la provincia de Zamora observamos una dualidad de opciones en las labores a realizar sobre el barbecho. En un primer grupo aparecen los fueros de Zamora y con dudas los de Cañizo, Santa Cristina y Villalonso (Fig. 2). En un segundo, encontramos los de Valle, Fuentesaúco, Fresno de la Ribera, la segunda redacción del de Santa Cristina, Fradejas, Almaraz de Duero y Bamba (Fig. 3) ${ }^{30}$.

kada un anno, unn a sembrar $e$ otro a trillar, con cuerpos, $e$ con bues, $e$ con bestias; el qui ouiere jugu de bues o de bestias, ajude con ell, e el qui ouiere un bue do bestia, ajude con ell. El texto lo recoge Juan José GARCÍA GonZÁlEZ, «Rentas de trabajo en San Salvador de Oña: Las sernas (1011-1550)», Cuadernos Burgaleses de Historia Medieval, 1 (1984), 119-194, en nota 118, en la p. 173.

27 Antonio Álvarez Tejedor, Estudio linguístico del léxico rural de la zona este de la provincia de Zamora (Salamanca: Universidad de Salamanca, 1989), 266 pp., los datos están en las pp. 54-57. Según este autor, las labores del barbecho varían en su zona de estudio - que comprende Tierra de Campos, Tierra del Pan y Tierra del Vinosiendo lo normal que se den tres rejas, en las dos primeras comarcas citadas, y de seis a ocho en la tercera. Además de los términos mencionados incluye otros, que no se relacionan con los medievales, así como la presencia de aquellos en pueblos y comarcas del área leonesa.

28 Juan Carlos GonZÁleZ Ferrero, Sociolingüística y variación dialectal. Estudio del habla de Flores de Aliste (Zamora: Instituto de Estudios Zamoranos «Florián de Ocampo». Excma. Diputación Provincial de Zamora, 1986), 214 pp., en pp. 428 y 429. En el libro de José María BAZ, El habla de la tierra de Aliste (Madrid: CSIC, 1967), 149 pp., se mencionan los términos rielva, para designar a la primera vuelta, y bimar y terciar para las siguientes.

29 Julio BoRREgo NIETO, Sociolingüística rural. Investigación en Villadepera de Sayago (Salamanca: Universidad de Salamanca, 1981), 383 pp., en pp. 81 y 82.

30 José Luis MARTIN RodRíguez en op. cit. en segundo lugar en la nota 23, en p. 16, menciona que en Villamor de los Escuderos, los vasallos del obispo de Zamora que tuvieran bueyes debían alzar, binar, terciar y sembrar. Los textos que recoge J. RODRíGUEZ FERNÁNDEZ, op. cit. en nota 2, pp. 364 y 370-371, correspondientes a los años 1257 y 1259 , no incluyen estas labores. 


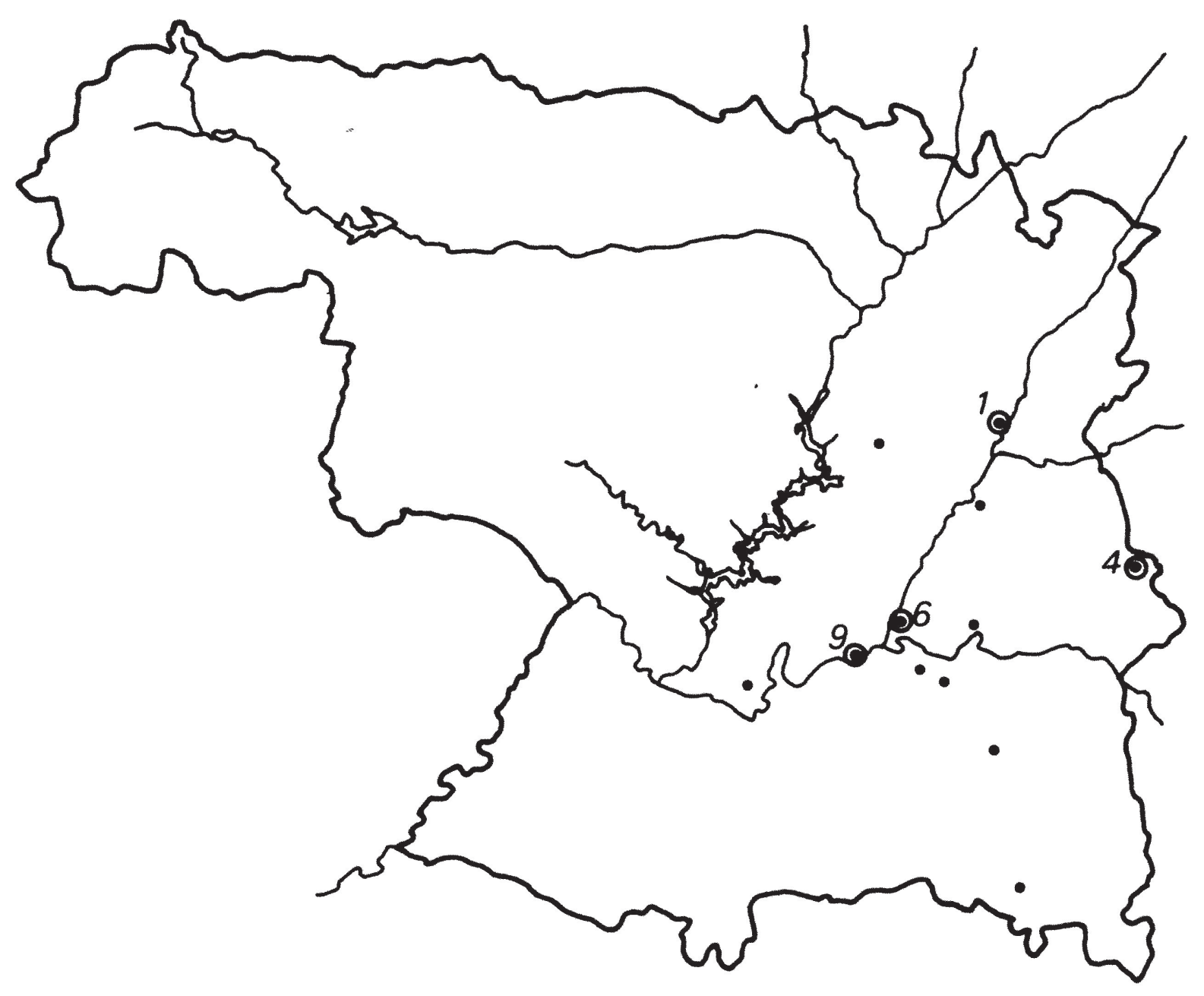

Fig. 2. Lugares en los que se constata la presencia de tres rejas, o más, en el barbecho, previas a la siembra. La numeración de las localidades se corresponde con la enunciada en la figura 1. 


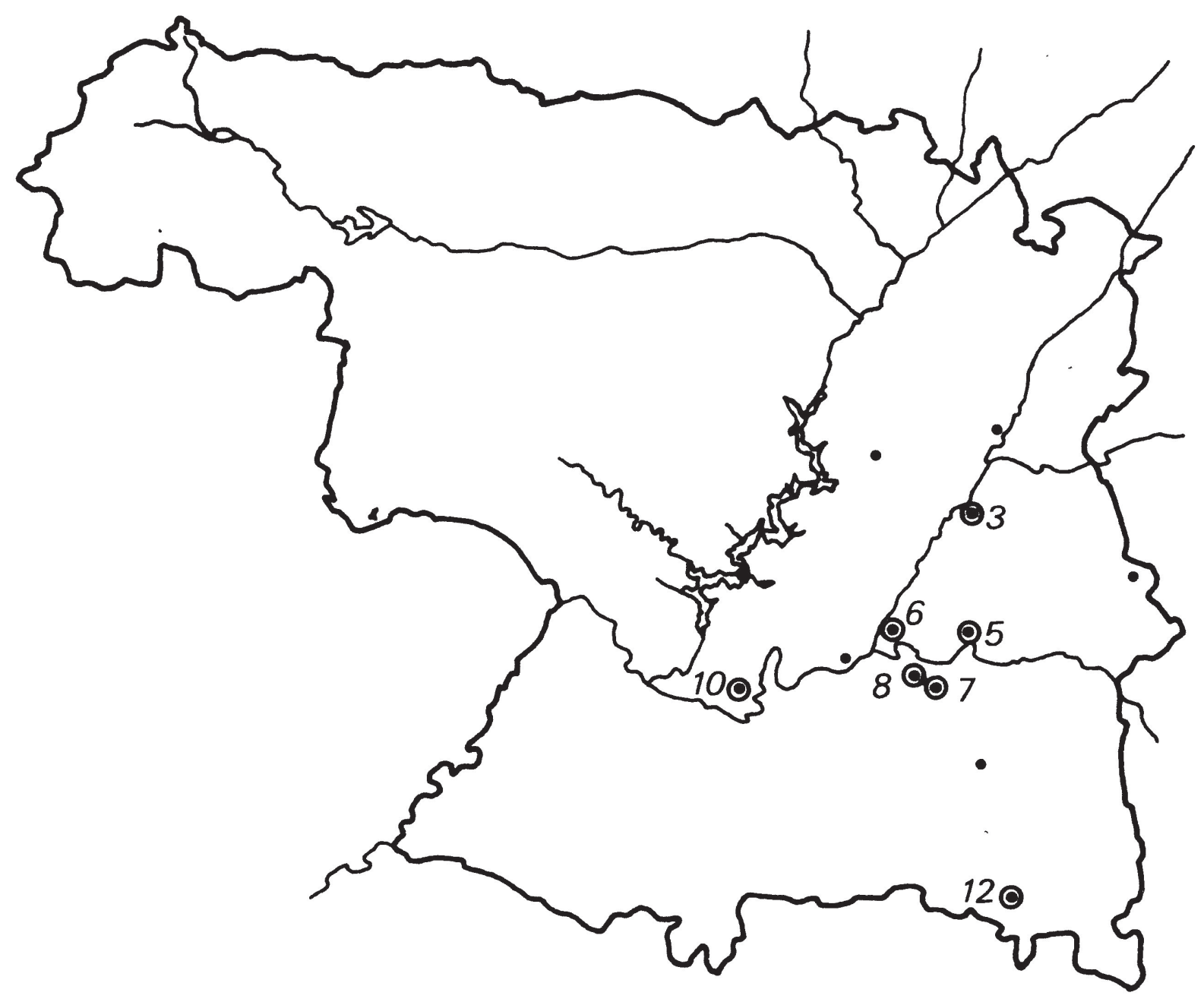

Fici. 3. Lugares en los que se constata la existencia de dos rejas en el barbecho, previas a la siembra. Se excluyen los representados en la figura 2, salvo Santa Cristina. La numeración de las localidades se corresponde con la enunciada en la figura 1. 
Entre los primeros destaca el de la capital zamorana por su claridad. Al recoger los trabajos que debe de hacer el yuguero ${ }^{31}$ en relación con el cultivo del cereal; dice: faga con elos [los bueyes que le prestará el señor] bonos barvechos, relvados e bimados e terciados e quartados, e los de trigo $e$ os de ordio. Frase que, como puede verse, podría considerarse la síntesis escueta del largo texto de Ibn Bașşāl citado al comienzo de este artículo.

Por su parte, en el fuero de Cañizo se introduce una alusión a la costumbre, como manera de regular las aradas, dando la impresión de que lo que se busca es el trabajo bien hecho independientemente del número de rejas a dar. Así, los hombres que tengan bueyes deben ir todos los años ad nostram sernam ralvare per duos dies cum suis bobus; et quantum laboraverint in ipsis duobus dies debent arare per duas vel tres vizes vel plus, secundum usum terre, seminare... No quedando demasiado claro si esa repetición de rejas debe hacerse en los dos días de la ralba o con posterioridad a la misma.

Frente al detalle del fuero de Zamora, en el fuero de Santa Cristina sólo se cita el número de sernas a realizar: a senrra, a barveiros, III ieras, et a seminare ${ }^{32}$. No obstante, en el nuevo fuero que se concede a esta localidad por parte de Alfonso IX en 1226, únicamente se especifica que relevent, et bimient et seminent, con lo cual no hay forma de saber si se ha producido un cambio en la técnica de cultivo en relación con la eliminación de una serna -el terciar el barbecho- o si esa tercera serna se empleaba en otras de las labores que aparecen detalladas en el texto del siglo XIII, es decir: segar, acarrear y trillar.

Tampoco en el fuero de Villalonso se aclara en qué se empleaban las cuatro sernas que debían dar, ya que sólo se dice que faciant IIII ieras in

31 Sobre la figura del yuguero se puede consultar el artículo de Juan Carlos MARTín CEA, «Una pequeña contribución al conocimiento del campesinado castellano: el yuguero», en El pasado bistórico de Castilla y León. Volumen 1. Edad Media -citado en la nota 25-, pp. 101-112 y el de Manuel RIU, «Agricultura y ganadería en el Fuero de Cuenca», Anuario de Estudios Medievales, 12 (1982), 167-181, en p. 172, quien anota como obligaciones del yuguero, relacionadas con faenas agrícolas, el arar, estercolar, desterronar, segar, trillar, recoger la paja y aventar el grano con su señor.

32 La palabra iera es sinónimo de serna y ha perdurado, con otro significado, hasta nuestros días. Como hemos señalado en nuestro trabajo citado en la nota 1 , en nota 10 en p. 416, este término aparece también en la provincia de León. Esperanza Botella Pомво en La serna: Ocupación, organización y explotación del espacio en la Edad Media (800-1250) (Santander: Tantín, 1988), no recoge esta voz al analizar las diversas denominaciones de la prestación laboral agraria en su zona de estudio, que comprende el espacio incluido entre el mar Cantábrico y los ríos Arlanzón, Pisuerga y Bayas. Esta autora pasa revista a los términos: servicio, opera, labor, obrero, facendera y serna. 
arada. El que hagan referencia al barbecho no deja de ser una suposición, aunque tenga cierta lógica ${ }^{33}$.

Como se ve, en este grupo se contemplan dos situaciones jurídicas diferentes ${ }^{34}$. Por un lado, está la relación contractual de la yuguería, que bien podría estar reflejando una realidad generalizada en cuanto a técnicas de cultivo se refiere. Cabría suponer que al yuguero se le exija lo que es normal en la zona, el secundum usum terre del fuero de Cañizo. Junto a esta faceta, en el resto de los fueros citados se está aludiendo a las sernas o prestaciones de trabajo obligatorio al señor en el régimen feudal, apareciendo la duda sobre si la supresión de algunas rejas se debe a que las jornadas de trabajo requeridas por el señor feudal se utilizan para otras labores o si, por el contrario, la no aparición de esa faenas de arada está reflejando realmente su inexistencia.

Esta última situación, la prestación de sernas, es la única que aparece en el segundo de los grupos mencionados más arriba y, a través de él, pensamos que se llega a intuir cómo la costumbre puede imponerse o generalizarse.

La idea de que una institución pueda imponer un sistema de cultivo es clara, en nuestra opinión. La reiteración de un modelo jurídico aplicable a varias localidades dominadas por el obispado así lo indica. La situación, por otra parte, no es exclusiva de tierras zamoranas, como puede comprobarse acudiendo al interesantísimo texto sobre las propiedades del cabildo segoviano que editó J. L. Martín. Son varias las veces que, en él, se dice de manera expresa, no deja lugar a dudas, que se debe trabajar de acuerdo a las normas del Cabildo. Así, por ejemplo, en el testimonio de Iohan Domínguez se señala:

Item ay XXXa III arençadas de viñas: las XXIII se deven labrar bien cada año a la costumbre del cabildo ${ }^{35}$.

En otros casos, como el de Estevan Blásquez, se expresa de otra manera:

e á las [viñas] de mantener de esta manera: que las escave e las pode e las cave cada año so la pena del cabildo ${ }^{36}$.

33 Como hemos visto -en nota 24 - las labores de arada pueden darse en el cultivo de la vid.

34 Con mayor claridad se puede apreciar este hecho consultando los encabezamientos de los textos en el Apéndice documental.

35 Op. cit. en nota 19, p. 112.

36 op. cit. en nota 19 , p. 146. Las condiciones no son exactamente iguales en todos los casos, lo que es fácil de entender si pensamos en la amplitud de las posesiones del Cabildo y que no todas ellas debían tener igual problemática. 
Volviendo a la provincia de Zamora, casi todos los fueros de este segundo grupo están otorgados por obispos de la sede zamorana que, en buena lógica, debían conocer perfectamente la reglamentación de su ciudad. El fuero de Valle y la segunda redacción del de Santa Cristina son los únicos no pertenecientes a la mitra zamorana; el primero está concedido por el conde Ramón de Borgoña y su esposa, la infanta Urraca Alfónsiz y el segundo por Alfonso IX, como se ha dicho. El obispo Bernardo concede los fueros de Fuentesaúco, Fresno de la Ribera y Fradejas, Martín I el de Almaraz de Duero y Martín II el de Bamba, y en todos ellos aparecen mencionadas de forma expresa las labores de arada. En ninguno, sin embargo, se cita que deban realizarse sobre el barbecho, pero no parece que quede otra posibilidad ya que el empleo de ralbar y binar precediendo a la labor de la siembra no deja lugar a muchas dudas.

El único texto de un señorío laico de este grupo obliga a que los $\mathrm{Ba}$ rones de Valle faciant illa serna de palacio II dies ad relvare, et bimalla, et seminalla. El texto real manda a los hombres de Santa Cristina y de su término que faciant sernas que fiunt in alfoz de Aradyn, et relevent, et binient, et seminent.

En el primero se los pueblos citados dependiente del obispado, se dice: Et homines de Fonte de Savugo faciant sernam ad suum Episcopum: Unum diem a relvar et alium ad bimar et tercium ad seminar. Lo que se repite en Fresno de la Ribera: Et faciant ad illum Episcopum sernas: Una die ad relvar et alia ad binar et alia seminar. En Fradejas, se recoge de forma similar: Et faciant ad illo Episcopo sua serna a relvar, alia a binar, alia a sembrar. En Almaraz de Duero se produce un incremento de labores en el fuero arzobispal, si se compara con el que en 1175 habían concedido a esta localidad Pedro Pérez y Fernando Cid. En el de 1175, sólo se debía dar al señor un día de arada ${ }^{37}$. El obispo Martín I al conceder el nuevo fuero, retoma la costumbre arzobispal y obliga a que las sernas se hagan boc modo: Una die a rebrar, alia a bimar, alia a seminare. Por último, el fuero de Bamba vuelve a ser una repetición de los anteriores: Dent in serna Episcopi, domini, sui, duos dies a relvar. Et illud quod relvaverint, biment, seminent..., donde sólo varía el incremento de un día que recibe

37 Junto a este día de arar, deben segar, trillar o majar y ayudar a acarrear el grano limpio. Estas obligaciones varían en el nuevo fuero, ya que además de ralbar, binar y sembrar, deben segar, acarrear a la era y terere (?) et ducere el pan a Zamora. Donde ese terere quizá sea un trabere con el que poner más énfasis en el hecho de llevar el grano hasta las dependencias del obispo, o, posiblemente haya que interpretarlo como una alusión a la faena de trilla. 
la primera reja y que coincide, en este aspecto, con los dos días que también ocupaba ésta en los fueros de Valle y de Cañizo ${ }^{38}$.

A la hora de buscar una explicación que ilustre el porqué de la diferencia en el número de rejas dadas al barbecho cabe excluir ciertas causas y recurrir a otras.

Si admitimos que el fuero de Cañizo indica que deben darse cuatro o más rejas, tendríamos que eliminar la hipótesis de que se produce un cambio de técnicas de cultivo a la largo del tiempo, ya que éste y el fuero de Zamora son los que cierran y abren, temporalmente, los textos reunidos aquí. Si, por el contrario, se opina que en Cañizo sólo se da la primera reja (con varias pasadas) el ámbito cronológico del primer grupo se reduce algo, abarcando desde mediados del siglo XI hasta mediados del siglo XII. Por lo que respecta al segundo grupo, la primera mención aparece a finales del XI, la de Valle, y la última es del inicio del segundo cuarto del XIII.

Más clara parece la distribución por donantes, mostrando, en el caso de la sede zamorana, bastante homogeneidad, como se ha dicho. El resto de los fueros no permite hallar una tendencia común en las técnicas agrícolas, quizá por su escaso número.

No se puede descartar el que estos textos estén reflejando simplemente una serie de variantes locales, sin más, o que la situación de los dominios señoriales y arzobispales imponga unas sernas más ligeras en las labores de arada ${ }^{39}$. Pero, parece más lógico pensar que existe otro motivo y, posiblemente, el texto de Ibn Bașșāl citado al comienzo de este artículo pueda venir en nuestra ayuda. Los fueros del segundo grupo encajan perfectamente en la apostilla que hace el autor toledano al final de la descripción de las labores que deben hacerse. Tomando la traducción medieval castellana, a la tierra cumple que la labren dos veses quando fuere buena tierra ${ }^{40}$. Quizá sea esa condición de buena tierra la que aparece en estas posesiones y la

38 J. L. MARTín Rodrícuez, op. cit. en primer lugar en nota 23, p. 14, deduce de este texto que «cada vasallo de Bamba se ocupaba de todas las faenas agrícolas en una zona determinada». No creemos que pueda sacarse esa conclusión, ya que lo que el texto indica es que «todo aquello» que se ralbe, debe binarse, sembrarse, etc., pero no que exista una correlación entre la tierra trabajada y la persona que lo hace.

39 Para ver si existe esta disminución de las cargas en los señoríos eclesiásticos y laicos habría que realizar un estudio comparativo entre éstos y los de realengo, de todos los tipos de cargas y no sólo de las sernas. Estudio que queda lejos de nuestra especialización científica y, pensamos que plantearía ciertos problemas a la hora de encontrar baremos de homologación entre cargas de distinto tipo. No parece demasiado factible, sin embargo, que esta situación se produzca, si nos atenemos al caso citado de Almaraz de Duero o si acudimos al de Villalonso. En cualquier caso, la variedad existente es amplia, si exceptuamos lo dicho sobre el obispado.

\footnotetext{
$40 \quad$ Ver nota 9.
} 
que permite, por tanto, reducir el número de rejas ${ }^{41}$. La hipótesis parece factible, aunque sea sólo eso: una hipótesis.

\section{José LUIS MINGOTE CALDERÓN}

Museo Arqueológico Provincial de Palencia

Con este breve artículo nos acercamos de nuevo a la documentación medieval para llevar a cabo una lectura etnológica de la misma. Esta vez nos centramos en los fueros de la provincia de Zamora para ver la incidencia de determinado colectivo, en concreto el obispado de la capital, en la implantación de técnicas agrarias relacionadas con el barbecho. Además, mostramos cómo el mundo cristiano aparece fuertemente relacionado con el musulmán en este tema concreto.

In this paper we study again the medieval documentation for reading it from an ethnological point of view. This time we analyse the fueros of the Province of Zamora to look into the incidence of bishopric of the capital on the implantation of agricultural techniques related with the fallow land. Moreover, we show how the medieval christian society in Spain appears strongly related with the Islamic one in this subject.

41 El texto árabe menciona, como hemos visto, lo innecesario de rebasar las dos labores si «la tierra fuera de buena calidad». 


\section{APÉNDICE DOCUMENTAL 42}

Fuero de Zamora, ant. a 1062 (Doc. 1, pp. 249-267).

De jugariis

57. Yuguero a quien dieren bues sanos e guaridos e IX caradas de pan e cada semana una ochava de farina para so beverayo, para VI meses, faga con elos bonos barvechos, relvados e bimados e terciados e quartados, e los de trigo e los de ordio, hu caban $\mathrm{X}$ fanegas de pam semradura; e se demays fezier, semrelos juguero de son pan. E barvechos de bima non sean devengados; e se esto non fezier, pierda ela nafaga e elos barvechos e quanto le dar deven.

58. ... E el iuguero que fizier ela sementera, faga elos barvechos assí como de suso son dichos, quartados para $\mathrm{X}$ fanegas senbradura de trigo e de cevada; e se los non fezier, pierda el quarto e elos barvechos, e estensse a cossimiento de so sennor.

\section{De vendicion de bues}

60. Omne que vendir boys commo vezino a vezino, tengalos ata IX dias. E se buenos fueren darada, tengalos, e enno tienpo de las miesses, pruevelo al carro e enna trilla. E se non fueren bonos, tornelos a los IX dias a so duenno e so duenno tornele so aver; e se ye so aver non le quisier dar, use elos boes e prinde por so aver. E se IX dias fueren pasados, non ye responda.

Fuero de Santa Cristina. 1062, Febrero, 1. Otorgado por Fernando I (Doc. 2, p. 268).

2. Et facendeira, a senrra, a barveiros, III ieras, et a seminare, quantum ubi arent et darent ad illos pane et vino et carne, ...

Fuero de Valle. 1094. Concedido por el conde Ramón de Borgoña y su esposa la infanta Urraca Alfónsiz (Doc. 4, p. 270).

42 El número de documento, las páginas y los números de epígrafe, hace referencia a los del libro de J. RodrígueZ FernÁndeZ, citado en la nota 2. 
4. Barones de Valle faciant illa serna de palacio II dies ad relvare, et bimalla, et seminalla, ...

4

Fuero de Venialbo. 1126, Mayo, 6. Concedido por el abad Pedro y su convento de Santo Tomé (Doc. 5, pp. 271-272).

13. Qui primo araverit et alio venerit super eum, ipso qui primo araverit faciat ipsa iusticia quomodo iudicamus: Quanto potuit iactare sua aguilada de sua manu et ponat ibi suo marcu et laboret altro qui venerit.

14. Dicimus de nostros foros de Sancti Thome: In primis, antequam partant, prendamus duas sernas ubi arent nostros boves, et nostro orto et nostra era et nostro Monasterio cum LX pasales.

16. Et omnes qui in ipso cauto laboraverint, de ipso que laboraverint in ipsa hereditate de Venialvo cum suos terminos, de ipso labore que ibi laboraverint cum bove vel cum azada, donent inde decimas vel primicias Deo et Sancti Thome apostoli.

Fuero de Fuentesaúco. 1133, Abril, 23. Otorgado por el obispo Bernardo, de Zamora (Doc. 7, p. 275).

3. Et homines de Fonte de Savugo faciant sernam ad suum Episcopum: Unum diem a relvar et alium ad bimar et tercium ad seminar.

Fuero de Fresno de la Ribera. 1146, Enero, 1. Otorgado por el obispo Bernardo, de Zamora (Doc. 9, pp. 278-279).

1. ... Et faciant ad illum Episcopum sernas: Una die ad relvar et alia ad binar et alia seminar, ...

3. Et de quantum laboraverint cum boves et cum azada, id est, de omni labore eorum extra ervos, dent decimas ad ecclesiam que fuerit in Frexino.

Fuero de Villalonso. 1147? Dado por los condes Osorio Martínez y su mujer Teresa Fernández (Doc. 10, pp. 280-281). [Concedido también a Benafarces (Valladolid)]. 
1. Ut faciant IIII ieras in arada in quantum potuerint confectare, et in ipsos quatuor dies de illa serna panem et vinum quantum illis abundet. Et qui non habuerit bove det suo asino, si habuerit, que ipso die vadat et veniat, et colant illum in facienda. Et qui non habuerit bovem neque asinum faciat secundum possibilitatem suam.

Carta de población de Fradejas. 1148, Agosto, 8. Dado por el obispo Bernardo, de Zamora (Doc. 11, pp. 282-283).

4. Et faciant ad illo episcopo sua serna a relvar, alia a binar, alia a sembrar, ...

10. Et de omnibus qui laboraverint cum boves et cum axada, id est, de omni labore, et dent suas decimas ad ecclesia que fuerit in Fradexes.

Fuero de Almaraz de Duero. 1175, Octubre, 1. Dado por Pedro Pérez y Fernando Cid (Doc. 20, pp. 294-295).

2. Et dar al sennor al dia de arar: a la iantar, pan et vino et casec; et ad illa cena, pan et vino et conducto.

Fuero de Almaraz de Duero. 1194-1217. Dado por el obispo Martín I, de Zamora (Doc. 38, p. 324).

1. Ut predicti homines de Almaraz faciant ei senram de XXti IIIIor cortes que sunt in rengalengo quod fuit domni Regis, hoc modo: Una die in rebrar, alia in bimar, alia in semimare...

Concierto habido entre la iglesia de Zamora y Guterre Pardo, sobre la contienda surgida entre ambas partes por razón de una heredad que poseía el segundo como vasallo de la iglesia, en Almaraz. 1224, Marzo, 20 (Doc. 46, pp. 337-338).

1. ... et illis [cortes] quos in eis posuerint sint vasalli inmediate ipsorum, ipsi autem sint vasalli Episcopi et faciant sernam cum quatuor iugis boum et dimidium...

2. ... Et de illis IIIIor cortibus et dimida, quas ibi habent predictus G. et uxor sua M., faciant ipsi vassalli Episcopo suam sernam cum IIIIor iugis boum... 
Fuero de Bamba. 1224, Junio. Dado por el obispo Martín II, de Zamora (Doc. 48, pp. 340-341).

2. Dent in serna Episcopi, domini sui, duos dies a relvar. Et illud quod relvaverint, biment, seminent, ...

3. Et istud faciant de bono labore ubi Episcopus voluerit in termino de Bamba et quando voluerit et cum melioribus bobus quos habuerint. Ita quod si habuerit iuga vel amplius in Bamba vel in suo termino, det istam sernam duos boves tantum.

4. Qui vero iugariam non habuerit et bovem habuerit faciat sernam cum alio bove quem sibi dederit maiorinus Episcopi. Si vero bovem non habuerit, faciat sernam cum suo corpore ubi maiorinus Episcopi mandaverit in Bamba vel in suo termino.

5. Si tota iuga bovum deffuerint quod serna non sit tota relvata, illi qui invitati ex parte episcopi fuerint et relvare non iverint, quotquot dies neglexerint, quilibet eorum duos solidos et quatuor denarios Episcopo, domino suo, pectet quousque relvet per duos dies.

6. Si autem per illos qui iverint tota serna fuit relvata, illi qui invitati ire noluerint, unusquisque pectet parti Episcopi duos solidos et quatuor denarios pro unaquaque die et faciat alias sernas cum Concilio. De quibus, quotquot neglexierit, det duos solidos et quatuor denarios pro unaquaque die pectet Concilio.

8. Quando sernam relvaverint, bimaverint et seminaverint, det eis Episcopus: in mane, panem, vinum et caseum; et in sero, panem vinum et carnem.

Nuevo fuero de Santa Cristina. 1226, Febrero, 27. Dado por Alfonso IX (Doc. 49, pp. 342-343).

2. Homines de Sancta Christina et de suo termino faciant sernas que fiunt in alfoz de Aradayn, et relevent, et binient, et seminent, ...

5. Quicumque etiam laboraverint in Sancta Christina et in suo termino, cum quantis iugis boum laboraverint, cum tantis faciant sernas supradictas.

Fuero de Fradejas. 1232, Novembre. Dado por el obispo Martín, de Zamora (Doc. 52, p. 348).

1. ... sub tali conditione ut omnes qui ibi populauerint sint absoluti a roxo, a nuncio, a serna, ... 
Fuero de Cañizo. 1234, Diciembre, 28. Dado por el abad Domingo, de Carracedo (Doc. 53, pp. 349-350).

2. Deinde, omnes habentes casas, ortos et labores boum dent forum et prandium abbatis vel prioris vel cellerarii integrum, scilicet, IIII solidos. Non habentes vero labores boum, dent forum et prandium medium, scilicet duos solidos.

7. Omnes haventes boves vadant annuatim ad nostram sernam ralvare per duos dies cum suis bobus; et quantum laboraverint in ipsis duobus diebus debent arare per duas vel tres vizes vel plus, secundum usum terre, seminare, [...] Non haventes boves vadant ad vineam cum suis legonibus, vel ad aliud servitium quod ex parte nostra illis fuerint commendatum.

8. Si quis illorum ad sernam levaberit jugum vel aratrum vel comerem [(sic) por vomerem] malum, vel non compleverit bene laborem suum, vel ibi rixas permoverit, vel aliquio malum fecerit, in alio die laborem suum compleat et unum solidum nobis pectet.

Concierto entre el obispo de Zamora y el concejo de Manganeses de Lampreana sobre la explotación de las tierras labrantías y del viñedo. Siglo XIII (Doc. 77, II, p. 387).

1. Todo omne que ovier valía de L. $^{2}$ moravedis sea tenudo de poner un iugo de boys en arada. E quien ovier valía de XXX moravedis ponga un boy con otro omne que ponga otro en arada. Et quien lo non feziere, peche $\mathrm{V}$ moravedis.

2. Otrossi, quien ovier vinna et la non serrare de guisa que non venga por ella danno a los vizinos, et la non lavrar de quatro lavores al menos, escavar et podar, arar et acobondrar, que peche $\mathrm{V}$ moravedis. 